\title{
Sarcopenia correlates with systemic inflammation in COPD
}

This article was published in the following Dove Press journal:

International Journal of COPD

20 February 2017

Number of times this article has been viewed

\section{Min Kwang Byun' \\ Eun $\mathrm{Na} \mathrm{Cho}^{\prime}$ \\ Joon Chang ${ }^{2}$ \\ Chul Min Ahn' \\ Hyung Jung Kim'}

'Division of Pulmonology, Department of Internal Medicine, Gangnam Severance Hospital, ${ }^{2}$ Division of Pulmonology, Department of Internal Medicine, Severance Hospital, Yonsei University College of Medicine, Seoul, South Korea
Correspondence: Hyung Jung Kim Division of Pulmonology, Department of Internal Medicine, Gangnam Severance Hospital, Yonsei University College of Medicine, 2II Eonju-ro, Dogok-dong, Gangnam-gu, Seoul 06273, South Korea Tel +82220193316

Fax +82234633882

Email khj57@yuhs.ac
Background: Muscle wasting and chronic inflammation are predominant features of patients with COPD. Systemic inflammation is associated with an accelerated decline in lung function. In this study, the prevalence of sarcopenia and the relationships between sarcopenia and systemic inflammations in patients with stable COPD were investigated.

Materials and methods: In a cross-sectional design, muscle strength and muscle mass were measured by handgrip strength (HGS) and bioelectrical impedance analysis in 80 patients with stable COPD. Patients ( $\geq 40$ years old) diagnosed with COPD were recruited from outpatient clinics, and then COPD stages were classified. Sarcopenia was defined as the presence of both low muscle strength (by HGS) and low muscle mass (skeletal muscle mass index [SMMI]). Levels of circulating inflammatory biomarkers (IL-6 and high-sensitivity TNF $\alpha$ [hsTNF $\alpha]$ ) were measured.

Results: Sarcopenia was prevalent in 20 (25\%) patients. Patients with sarcopenia were older, had lower body mass index, and a higher percentage of cardiovascular diseases. In addition, they had significantly higher modified Medical Research Council scores and lower 6-minute walk distance than those without sarcopenia. HGS was significantly correlated with age, modified Medical Research Council score, and COPD Assessment Test scores. Both HGS and SMMI had associations with IL-6 and hsTNF $\alpha$ (HGS, $r=-0.35, P=0.002$; SMMI, $r=-0.246, P=0.044$ ) level. In multivariate analysis, old age, lower body mass index, presence of cardiovascular comorbidities, and higher hsTNF $\alpha$ levels were significant determinants for sarcopenia in patients with stable COPD.

Conclusion: Sarcopenia is very common in patients with stable COPD, and is associated with more severe dyspnea-scale scores and lower exercise tolerance. Systemic inflammation could be an important contributor to sarcopenia in the stable COPD population.

Keywords: sarcopenia, muscle wasting, handgrip strength, systemic inflammation, COPD

\section{Introduction}

COPD is a leading cause of morbidity and mortality worldwide. It is characterized by persistent, progressive limitations in airflow, which are usually associated with enhanced airway and lung-inflammatory responses to noxious particles or gases. ${ }^{1}$ Systemic inflammation is associated with an accelerated decline in lung function, and is heightened during episodes of exacerbation., ${ }^{2,3}$ Systemic inflammation is now a recognized risk factor for other complications commonly observed in patients with COPD, including atherosclerosis, ${ }^{4}$ cachexia,${ }^{5}$ anorexia, ${ }^{6}$ and osteoporosis. ${ }^{7}$ It is present not only in acutely exacerbated COPD but also in stable COPD. ${ }^{8}$

Muscle wasting is another characteristic feature of COPD, ${ }^{9}$ and evaluation of both muscle quality and quantity are essential. Physical inactivity is involved in the early loss of muscle function in COPD, and muscle wasting can further decrease physical 
activity, exercise tolerance, and quality of life. Previous studies have reported that muscle wasting is closely associated with higher rates of COPD exacerbation and lower survival rates. ${ }^{10,11}$ The BODE index (body mass index [BMI], obstruction of airways as measured by forced expiratory volume in 1 second $\left[\mathrm{FEV}_{1}\right]$, dyspnea as measured by the modified Medical Research Council [mMRC] dyspnea scale, and exercise capacity as measured by 6-minute walk distance [6MWD]) was originally designed to predict mortality in COPD. It also demonstrates the prognostic significance of muscle mass and exercise capacity (as assessed by BMI and 6MWD) in predicting long-term outcomes. ${ }^{12}$ This concept recently defined as sarcopenia a clinical syndrome comprised of multiple factors, including physical inactivity, malnutrition, inflammation, and chronic illness. ${ }^{13,14}$ The European Working Group on Sarcopenia in Older People (EWGSOP) recommends using the presence of both low muscle mass and low muscle function (strength or performance) for the diagnosis of sarcopenia. ${ }^{13}$

Initially, sarcopenia used to describe age-related loss of muscle mass and power. However, catabolic inflammatory processes often observed in chronic illnesses can enhance sarcopenia. As such, it has recently been recognized as a syndrome in various chronic conditions. Also, in the COPD population the prevalence of sarcopenia is reported to be $15 \%$, and is associated with impaired lung function and poor health status. No studies, however, have yet shown a direct association between sarcopenia and levels of inflammatory biomarkers in this group. We measured muscle mass and muscle strength in South Korean patients with stable COPD to determine the prevalence of sarcopenia using handgrip strength (HGS) and bioelectrical impedance analysis (BIA). In addition, we evaluated relationships between sarcopenia, biomarkers of systemic inflammation, disease severity, and other clinical features.

\section{Materials and methods}

\section{Study design and populations}

Patients ( $\geq 40$ years old) diagnosed with COPD according to the Global Initiative for Chronic Obstructive Lung Disease (GOLD) and attending outpatient clinics between June 2012 and June 2014 at Gangnam Severance Hospital were considered eligible. Investigators explained the aim and protocol of the study to patients who fulfilled the inclusion criteria. Written informed consent and medical history were obtained at the visit. This was a cross-sectional study, and all measurements were completed at a single visit for each participants. Exclusion criteria were age $<40$ years, presence of active lung disease, diagnosis of bronchial asthma, lung resection or transplantation, presence of severe cardiovascular disease, an episode of COPD exacerbation within the last month, inability to perform a 6MWD test due to severe dyspnea, lower-leg trauma, severe muscle weakness, and inability to read or understand informed consent documents. All consenting subjects completed a Korean version of the COPD Assessment Test (CAT) questionnaire (GlaxoSmithKline, London, UK). Subjects were evaluated using the mMRC dyspnea scale and postbronchodilator $\mathrm{FEV}_{1}$ in a pulmonary function test (Vmax 229; SensorMedics, Yorba Linda, CA, USA), 6MWD, muscle measurements, and venous blood sampling at a single visit. We evaluated history of exacerbations in the last year, the BODE index, and disease severity according to a 2011 GOLD revision; the revised document recommends assessment of symptoms, lung function, and risk of exacerbation. ${ }^{1}$

This study was performed in accordance with the Declaration of Helsinki and South Korean good clinical practice guidelines. All protocols were approved by the institutional review board of Gangnam Severance Hospital (3-2011-0300).

\section{Measurement of muscle mass and muscle strength}

Muscle mass was quantified with BIA (Body Composition Analysis 1000; MediGate, Seoul, South Korea). Height (cm), body weight $(\mathrm{kg})$, and BMI $\left(\mathrm{kg} / \mathrm{m}^{2}\right)$ were also measured. Fat mass index (FMI) and fat-free mass index (FFMI) were calculated as fat mass or fat-free mass (FFM) divided by the square of the patient's height $\left(\mathrm{kg} /\right.$ height $\left.^{2}\right)$. Skeletal muscle mass index (SMMI) was calculated as skeletal muscle mass (SMM) divided by the square of patient's height $\left(\mathrm{kg} /\right.$ height $\left.^{2}\right)$. Low MM was defined as having an SMMI at least two standard deviations (SDs) below normal sex-specific means in young persons. Data were compared with the 2008-2011 Korean National Health and Nutrition Examination Survey IV and V, a nationally representative survey conducted by the South Korean Ministry of Health and Welfare. To determine muscle strength, HGS was measured using a Jamar handheld dynamometer (Patterson Medical, Warrenville, IL, USA), the reliability of which has been previously established in community-dwelling older adults. Subjects were seated with shoulders at $0^{\circ}$ adduction and neutral rotation, elbows at $90^{\circ}$ flexion, and forearms in neutral position. Three trials were performed with a rest period of 1 minute between trials, and average values were recorded. Low muscle strength was defined as HGS values $\leq 30 \mathrm{~kg}$ in men and $\leq 20 \mathrm{~kg}$ in women. 
According to the recommendation of the EWGSOP, ${ }^{13}$ sarcopenia was defined when patients had both low MM (assessed by decreased SMMI lower than cutoff values) and low muscle strength (measured by low HGS values).

\section{Measurement of dyspnea and quality of life}

The mMRC dyspnea scale, which uses a $0-4$ scoring system, was used to assess shortness of breath. To assess functional status and exercise capacity, we used patients' $6 \mathrm{MWD},{ }^{15}$ which measures the distance that a patient can quickly walk along a corridor. Using these data, we measured patients' BODE index. The BODE index ranges from 0 to 10 points, with higher scores indicating a greater risk of death in COPD. ${ }^{12}$ To evaluate quality of life, we used the Korean version of the CAT, as previously mentioned. This questionnaire consists of eight questions scored on a $0-5$ scale.

\section{Measurement of biomarkers of systemic inflammation}

Venous blood was drawn from subjects, and circulating levels of biomarkers of systemic inflammation were measured using a high-sensitivity TNF $\alpha$ (hsTNF $\alpha$ ) enzyme-linked immunosorbent assay kit (Quantikine ${ }^{\circledR}$; R\&D Systems, Minneapolis, MN, USA) and a chemiluminescence assay to measure IL-6 (Immulite 2000; Siemens, Munich, Germany).

\section{Statistical analyses}

Statistical analyses were performed using SPSS software (version 23.0; IBM, Armonk, NY, USA). All data are expressed as means \pm SD or medians with interquartile ranges. The Kolmogorov-Smirnov test was used to analyze normality of distribution. For skewed data, including serum IL-6, natural $\log$ values were used. Continuous variables are described as means $\pm \mathrm{SD}$, and Student's $t$-test was used for comparative analysis. Categorical variables were analyzed using $\chi^{2}$ and $t$-tests with $95 \%$ confidence intervals (CIs). Pearson's correlation analysis was used to determine relationships among HGS, SMMI, and other clinical or inflammatory parameters. Multiple logistic regression analysis was performed to find significant determinants of sarcopenia. $P<0.05$ was deemed to indicate statistical significance.

\section{Results}

\section{Patient characteristics}

Table 1 shows 80 patients with stable COPD were included. Subjects were 68.4 \pm 8.9 (range 42-88) years old and $83.8 \%$ (67) were male. Sarcopenia was prevalent in $20(25 \%)$
Table I Baseline characteristics of study subjects

\begin{tabular}{|c|c|c|c|c|}
\hline Variable & $\begin{array}{l}\text { Total } \\
(\mathrm{n}=\mathbf{8 0})\end{array}$ & $\begin{array}{l}\text { With } \\
\text { sarcopenia } \\
(n=20,25 \%)\end{array}$ & $\begin{array}{l}\text { Without } \\
\text { sarcopenia } \\
(n=60,75 \%)\end{array}$ & $P$-value \\
\hline Age (years) & $68.4 \pm 8.9$ & $73.1 \pm 8$ & $66.8 \pm 8.7$ & 0.006 \\
\hline Male (\%) & $67(83.8)$ & $17(85)$ & $50(83.3)$ & 0.585 \\
\hline BMI $\left(\mathrm{kg} / \mathrm{m}^{2}\right)$ & $23.3 \pm 3.2$ & $21.1 \pm 1.7$ & $24.1 \pm 3.2$ & $<0.001$ \\
\hline \multicolumn{5}{|l|}{ Comorbidities (\%) } \\
\hline Diabetes & $20(25)$ & $6(30)$ & $14(23.3)$ & 0.374 \\
\hline Hypertension & $38(47.5)$ & $12(60)$ & $26(43.3)$ & 0.181 \\
\hline $\begin{array}{l}\text { Cardiovascular } \\
\text { disease }\end{array}$ & $19(23.8)$ & $10(50)$ & $9(15)$ & 0.003 \\
\hline Tuberculosis & $26(32.5)$ & $6(30)$ & $20(33.3)$ & 0.507 \\
\hline $\begin{array}{l}\text { Bronchiectasis/ } \\
\text { emphysema }\end{array}$ & $19(23.8)$ & $3(15)$ & $16(26.7)$ & 0.228 \\
\hline \multicolumn{5}{|c|}{ Body composition and strength } \\
\hline WHR & $0.92 \pm 0.04$ & $0.90 \pm 0.16$ & $0.91 \pm 0.05$ & 0.464 \\
\hline Fat mass (kg) & $15.7 \pm 5.3$ & $13.8 \pm 3.6$ & $16.4 \pm 5.6$ & 0.056 \\
\hline $\mathrm{FMI}\left(\mathrm{kg} / \mathrm{m}^{2}\right)$ & $5.8 \pm 1.9$ & $5.1 \pm 1.4$ & $6 \pm 2.1$ & 0.087 \\
\hline Body fat (\%) & $24.6 \pm 5.9$ & $24.1 \pm 5.1$ & $24.7 \pm 6.5$ & 0.729 \\
\hline $\mathrm{FFM}(\mathrm{kg})$ & $47.4 \pm 6.9$ & $42.8 \pm 4.6$ & $49 \pm 7.4$ & $<0.001$ \\
\hline FFMI $\left(\mathrm{kg} / \mathrm{m}^{2}\right)$ & $17.4 \pm 1.6$ & $\mid 5.9 \pm 1$ & $17.9 \pm 1.5$ & $<0.001$ \\
\hline SMM (kg) & $25.9 \pm 4.7$ & $22.8 \pm 3.5$ & $27 \pm 4.6$ & 0.001 \\
\hline SMMI $\left(\mathrm{kg} / \mathrm{m}^{2}\right)$ & $9.5 \pm 1.2$ & $8.5 \pm 0.8$ & $9.8 \pm 1.1$ & $<0.001$ \\
\hline HGS (kg) & $30.6 \pm 9.3$ & $25.4 \pm 6.1$ & $32.9 \pm 9.8$ & 0.002 \\
\hline \multicolumn{5}{|l|}{ Pulmonary function* } \\
\hline $\mathrm{FEV}_{1}(\%)$ & $61.2 \pm 14.3$ & $58 \pm 13.9$ & $62.3 \pm 13.9$ & 0.253 \\
\hline $\mathrm{FEV}_{1} / \mathrm{FVC}(\%)$ & $51 \pm 12.9$ & $46.1 \pm 13$ & $52.6 \pm 12.8$ & 0.05 \\
\hline \multicolumn{4}{|c|}{ GOLD stage, 201 I revised ${ }^{\#}(\%)$} & 0.624 \\
\hline A & $24(30)$ & $4(20)$ & $20(33.3)$ & \\
\hline B & $31(38.8)$ & $9(45)$ & $22(36.7)$ & \\
\hline $\mathrm{C}$ & $5(6.2)$ & $2(10)$ & $3(5)$ & \\
\hline $\mathrm{D}$ & $20(25)$ & $5(25)$ & $15(25)$ & \\
\hline
\end{tabular}

Notes: *Postbronchodilator; ${ }^{\# A c c o r d i n g ~ t o ~} 2011$ GOLD revision. Data expressed as mean \pm standard deviation or $\mathrm{n}(\%)$.

Abbreviations: BMI, body mass index; WHR, waist:hip ratio; FMI, fat mass index; FFM, fat-free mass; FFMI, FFM index; SMM, skeletal muscle mass; SMMI, SMM index; HGS, handgrip strength; FEV $\mathrm{l}_{1}$, forced expiratory volume in I second; FVC, forced vital capacity; GOLD, Global Initiative for Chronic Obstructive Lung Disease.

patients. Patients with sarcopenia were significantly older, showed lower BMI values, and had a higher percentage of cardiovascular disease. FFM, FFMI, SMM, and SMMI were markedly decreased in sarcopenic patients with BIA. HGS was also lower in patients with sarcopenia. There was no significant difference in COPD severity between patients with and without sarcopenia.

\section{Clinical outcomes associated with sarcopenia}

Table 2 shows that compared with patients without sarcopenia, patients with sarcopenia had significantly more severe symptoms of dyspnea and higher mMRC scores, but CAT scores were not significantly different between the two groups. These patients exhibited poorer exercise tolerance, as indicated by 
Table 2 Clinical outcomes according to presence or absence of sarcopenia

\begin{tabular}{llll}
\hline Variable & $\begin{array}{l}\text { With } \\
\text { sarcopenia } \\
(\mathbf{n}=\mathbf{2 0}, \mathbf{2 5} \%)\end{array}$ & $\begin{array}{l}\text { Without } \\
\text { sarcopenia } \\
(\mathbf{n}=\mathbf{6 0}, \mathbf{7 5} \%)\end{array}$ & P-value \\
\hline mMRC score & $0.95 \pm 0.86$ & $0.62 \pm 0.7 \mathrm{I}$ & 0.044 \\
$0-\mathrm{I}$ & $15(75 \%)$ & $5 \mathrm{I}(85 \%)$ & 0.059 \\
$2-4$ & $5(25 \%)$ & $9(15 \%)$ & \\
CAT score & $15(7-22)$ & $\mathrm{I} 4(8.75-21.25)$ & 0.678 \\
$\quad<10$ & $6(30)$ & $26(43.3)$ & 0.216 \\
$\geq 10$ & $14(70)$ & $34(56.7)$ & \\
BODE index & $3(0-6)$ & $\mathrm{I}(0-4)$ & $0.00 \mathrm{I}$ \\
6MWD (m) & $350.8 \pm 78$ & $389.3 \pm 66.9$ & 0.042 \\
Acute exacerbations & $9(45)$ & $22(36.7)$ & 0.343 \\
$\geq \mathrm{I} /$ year $(\mathrm{n})$ & & & \\
Inflammatory biomarkers & & & \\
$\quad$ IL-6* & $\mathrm{I} .36 \pm 0.8 \mathrm{I}$ & $0.64 \pm 0.69$ & 0.044 \\
hsTNF $\alpha^{*}$ & $0.72 \pm \mathrm{I} .0 \mathrm{I}$ & $-0.03 \pm \mathrm{I} .16$ & 0.013 \\
\hline
\end{tabular}

Notes: *Log-transformed value. All data expressed as mean \pm SD or $\mathrm{n}(\%)$ except for CAT score, BODE index, and number of acute exacerbation in the previous year (median with inter-quartile ranges).

Abbreviations: mMRC, modified Medical Research Council; CAT, COPD Assessment Test; 6MWD, 6-minute walk distance; hsTNF $\alpha$, high-sensitivity TNF $\alpha$; BODE, body (mass index), (airflow) obstruction, dyspnea, exercise (capacity); SD, standard deviation.

the shorter 6MWD values. The median score in the BODE index was also significantly higher in patients with sarcopenia. There was no significant difference in the frequency of acute exacerbation of COPD between the two groups. Among inflammatory biomarkers, hsTNF $\alpha(P=0.013)$ and IL-6 $(P=0.044)$ levels were significantly higher in patients with sarcopenia than patients without sarcopenia.

\section{Correlations among HGS, SMM, and inflammatory biomarkers}

As shown in Table 3, HGS showed positive correlations with FFMI, SMMI, and 6MWD and significant negative correlations with age, FMI, mMRC score, CAT scores, IL-6, and hsTNF $\alpha$; SMMI positively correlated with BMI, HGS, FFMI, and FMI, and negatively correlated with BODE index, IL-6, and hsTNF $\alpha$.

Figure 1 shows HGS $(r=-0.35, P=0.002)$ and SMMI $(r=0.246, P=0.044)$ levels were very closely associated with hsTNF $\alpha$ as a systemic inflammatory parameter. In addition, values for the BODE index tended to correlate with HGS and SMMI. However, $\mathrm{FEV}_{1}{ }^{16}$ did not correlate with HGS or SMMI in this study.

\section{Factors associated with sarcopenia in patients with COPD}

As shown in Table 4, cardiovascular disease, a common comorbidity in patients with COPD, was associated with the presence of sarcopenia. GOLD classification (revised in 2011)
Table 3 Correlations among HGS, SMMI, and inflammatory biomarkers

\begin{tabular}{|c|c|c|c|c|}
\hline \multirow[t]{2}{*}{ Variable } & \multicolumn{2}{|l|}{ HGS } & \multicolumn{2}{|l|}{ SMMI } \\
\hline & Coefficient & $P$-value & Coefficient & $P$-value \\
\hline Age & -0.415 & $<0.001$ & -0.074 & 0.527 \\
\hline BMI & 0.001 & 0.998 & 0.852 & $<0.001$ \\
\hline HGS & I & - & 0.427 & $<0.001$ \\
\hline FFMI & 0.346 & 0.002 & 0.99 & $<0.001$ \\
\hline FMI & -0.325 & 0.004 & 0.275 & 0.017 \\
\hline SMMI & 0.427 & $<0.001$ & I & - \\
\hline $\mathrm{FEV}_{1}^{*}$ & -0.103 & 0.367 & 0.018 & 0.88 \\
\hline $\mathrm{FEV}_{1} / \mathrm{FVC}^{*}$ & -0.13 & 0.367 & 0.079 & 0.498 \\
\hline mMRC score & -0.346 & 0.002 & -0.062 & 0.595 \\
\hline 6MWD & 0.44 & $<0.001$ & 0.015 & 0.899 \\
\hline BODE index & -0.216 & 0.045 & -0.257 & 0.026 \\
\hline CAT score & -0.236 & 0.048 & -0.132 & 0.275 \\
\hline IL-6 $6^{\#}$ & -0.273 & 0.018 & -0.227 & 0.048 \\
\hline hsTNF $\alpha^{\#}$ & -0.35 & 0.002 & -0.246 & 0.044 \\
\hline
\end{tabular}

Notes: *Postbronchodilator; \#log-transformed value.

Abbreviations: BMI, body mass index; HGS, handgrip strength; FFMI, fat-free mass index; FMI, fat mass index; SMMI, skeletal muscle mass index; $\mathrm{FEV}_{1}$, forced expiratory volume in I second; FVC, forced vital capacity; mMRC, modified Medical Research Council; 6MWD, 6-minute walk distance; CAT, COPD Assessment Test; hsTNF $\alpha$, high-sensitivity TNF $\alpha$; BODE, body (mass index), (airflow) obstruction, dyspnea, exercise (capacity).

was not associated with sarcopenia. As a systemic inflammatory biomarker, hsTNF $\alpha$ levels showed significant negative correlations with the presence of sarcopenia in our analysis. In multivariate analysis, significant determinants for the presence of sarcopenia in patients with stable COPD were old age (odds ratio [OR] 1.19, 95\% CI 1.03-1.36), low BMI (OR $0.52,95 \%$ CI $0.35-0.78$ ), presence of previous cardiovascular disease (OR 4.66, 95\% CI 1.01-18.31), and higher hsTNF $\alpha$ levels (OR 1.99, 95\% CI 1.04-3.81).

\section{Discussion}

To summarize, the prevalence of sarcopenia in South Korean patients with stable COPD was $25 \%$, which was consistent with previous studies reporting prevalence rates of $20 \%-40 \% \cdot{ }^{17-19}$ Patients with sarcopenia tended to be older, have a higher percentage of cardiovascular comorbidity, more severe mMRC dyspnea scores, higher BODE index scores, and lower exercise tolerance in the 6MWD than those without sarcopenia. Sarcopenia in male and female patients was $25.4 \%$ (17 of 67) and $23.1 \%$ (three of 13). There was no difference in sex. The proportion of male sex among COPD patients was far higher in our study. However, it was not different from other South Korean COPD data. According to the representative South Korean COPD cohort data of nearly 1,000 COPD patients, $91.2 \%$ were male, higher than our data. ${ }^{20}$

MM measured by SMMI correlated with BMI and bodycomposition parameters, whereas muscle strength assessed by HGS significantly correlated with mMRC dyspnea scale, 

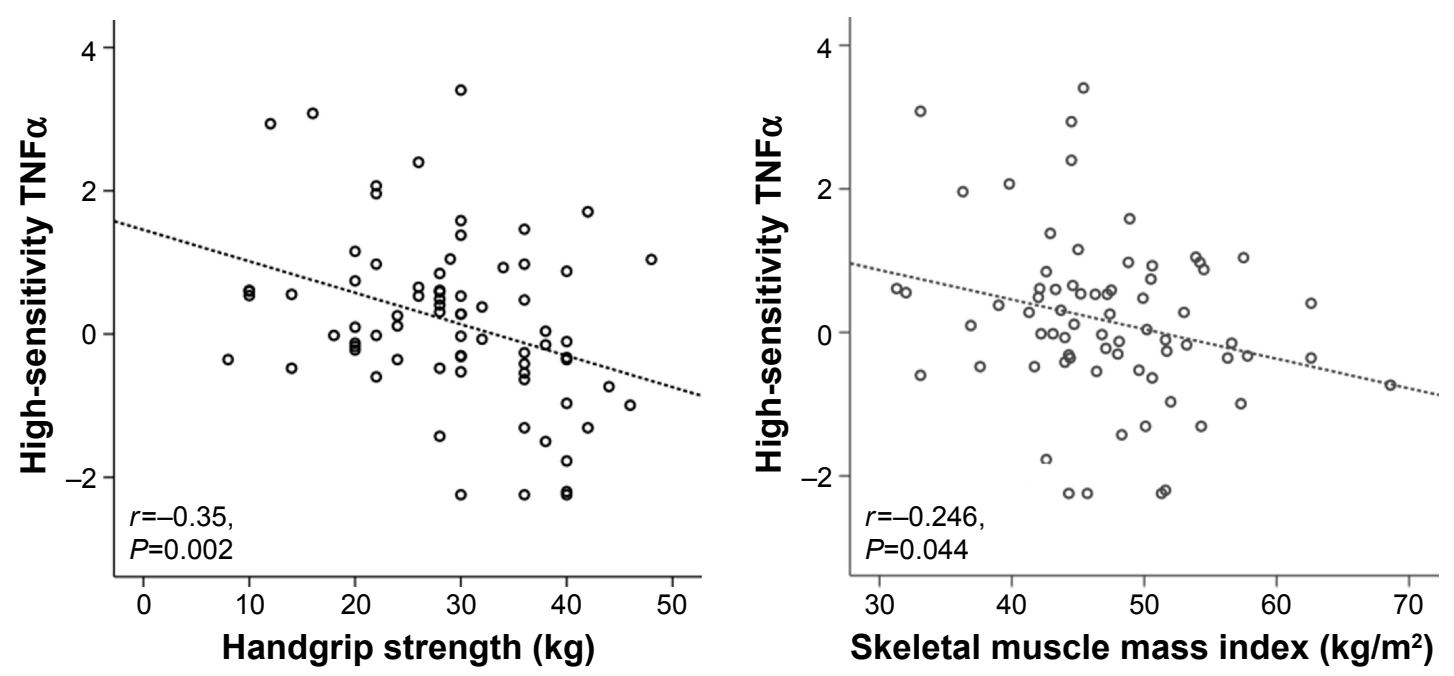

Figure I Correlation of handgrip strength and skeletal muscle mass index with high-sensitivity TNF $\alpha$. Abbreviation: TNF $\alpha$, Tumor necrosis factor alpha.

CAT score, and 6MWD as COPD clinical parameters. Low HGS and SMMI showed positive correlations with the BODE index. These results suggest sarcopenia is closely correlated with the BODE index, predicting higher risk of mortality. Consistent with our findings, Swallow et al reported that muscle strength is important in patient prognosis. ${ }^{21} \mathrm{~A}$ recent study of patients with stable COPD using prospective multicenter data also found that HGS correlated significantly with mortality. ${ }^{22}$ Therefore, HGS might provide critical prognostic information for patients with COPD.

In addition, the presence of sarcopenia was highly associated with levels of systemic inflammation. The mechanisms by which patients with COPD develop systemic inflammation

Table 4 Factors associated with sarcopenia

\begin{tabular}{|c|c|c|c|c|}
\hline \multirow[t]{3}{*}{ Variable } & \multicolumn{4}{|l|}{ Sarcopenia } \\
\hline & \multicolumn{2}{|c|}{ Univariate analysis } & \multicolumn{2}{|c|}{ Multivariate analysis } \\
\hline & OR (95\% CI) & P-value & OR (95\% CI) & P-value \\
\hline \multicolumn{5}{|l|}{ Age } \\
\hline Years & I.II (I.03-I.22) & 0.009 & $1.19(1.03-1.36)$ & 0.017 \\
\hline \multicolumn{5}{|l|}{ Sex } \\
\hline Male vs female & I.I3 (0.28-4.6) & 0.861 & - & - \\
\hline \multicolumn{5}{|l|}{ BMI } \\
\hline $\mathrm{kg} / \mathrm{m}^{2}$ & $0.63(0.48-0.82)$ & 0.001 & $0.52(0.35-0.78)$ & 0.001 \\
\hline \multicolumn{5}{|l|}{ Diabetes } \\
\hline Presence & $1.4 \mid(0.38-3.02)$ & 0.552 & - & - \\
\hline \multicolumn{5}{|c|}{ Cardiovascular disease } \\
\hline Presence & $5.66(1.8-12.65)$ & 0.003 & $4.66(1.01-18.31)$ & 0.048 \\
\hline \multicolumn{5}{|l|}{ GOLD stage } \\
\hline$A-B$ & I & & - & - \\
\hline$C-D$ & $1.26(0.43-3.67)$ & 0.676 & - & - \\
\hline hsTNF $\alpha^{*}$ & $1.81(1.1-2.99)$ & 0.019 & $1.99(1.04-3.81)$ & 0.038 \\
\hline
\end{tabular}

Notes: *Log-transformed value. Multivariate logistic analysis was performed after adjusting for age, sex, BMI, and diabetes.

Abbreviations: OR, odds ratio; $\mathrm{Cl}$, confidence interval; $\mathrm{BMI}$, body mass index; GOLD, Global Initiative for Chronic Obstructive Lung Disease; hsTNF $\alpha$, high-sensitivity TNF $\alpha$. are currently unknown. The presence of systemic inflammation, however, is closely associated with complications that affect prognosis, such as weight loss, ${ }^{23,24}$ cachexia, ${ }^{25,26}$ and cardiovascular disease. ${ }^{27,28}$ In a review of inflammatory markers in patients with COPD, CRP, fibrinogen, leukocytes, TNF $\alpha$, IL-6, and IL-8 levels were significantly elevated in patients with COPD compared with healthy controls. ${ }^{8}$ In this study, we assessed correlations of sarcopenia with the systemic inflammatory biomarkers IL-6 and hsTNF $\alpha$. Muscle strength assessed by HGS and MM measured by SMMI showed significant correlations with levels of IL-6 and hsTNF $\alpha$. In multivariate analysis, higher hsTNF $\alpha$ was a significant determinant for the presence of sarcopenia. The strength of this study was that most of patients in our study had milder COPD severity (GOLD A/B, 68.8\%) and all patients had stable status, not exacerbated, and significant correlations of HGS and SMMI with levels of systemic inflammatory biomarkers is a noteworthy result.

In the elderly and patients with COPD, physical inactivity is a key factor in the development of sarcopenia and a predictor of mortality and disability. ${ }^{29}$ The causes and prevalence of systemic inflammation and the mechanisms by which it leads to muscle wasting in COPD patients are uncertain. Previous studies have suggested that severe muscle wasting can lead to cachexia during systemic inflammation. ${ }^{24,30}$ We found that various factors were associated with sarcopenia, including age, BMI, and systemic inflammation. COPD severity, however, was not associated with the presence or absence of sarcopenia in this study, and the frequency of acute exacerbations of COPD was not different between the two groups. Considering the clinical complexity of COPD, however, we propose that multiple biomarkers are required 
to characterize pathogenic factors and clinical outcomes in this disease. ${ }^{31}$

There are several limitations to our study. We utilized a cross-sectional design, the study population was small, and recruitment was limited to outpatient clinics at a single tertiary hospital. Sample composition in terms of disease severity according to GOLD stage and $\mathrm{FEV}_{1}$ was not well balanced.

\section{Conclusion}

Nearly a quarter of South Korean patients with stable COPD have sarcopenia, and sarcopenic patients presented with more severe dyspnea, showed lower exercise tolerance, and predicted poor prognosis. In addition, sarcopenia was highly associated with levels of systemic inflammation. The presence of systemic inflammation was a main determinant of sarcopenia in a stable COPD population.

\section{Acknowledgment}

The authors are grateful for statistical support provided by the Medical Research Supporting Section of Yonsei University College of Medicine.

\section{Disclosure}

The authors report no conflicts of interest in this work.

\section{References}

1. Vestbo J, Hurd SS, Agustí AG, et al. Global strategy for the diagnosis, management, and prevention of chronic obstructive pulmonary disease: GOLD executive summary. Am J Respir Crit Care Med. 2013;187(4): 347-365.

2. Donaldson GC, Seemungal TA, Patel IS, et al. Airway and systemic inflammation and decline in lung function in patients with COPD. Chest. 2005;128(4):1995-2004.

3. Hurst JR, Donaldson GC, Perera WR, et al. Use of plasma biomarkers at exacerbation of chronic obstructive pulmonary disease. Am J Respir Crit Care Med. 2006;174(8):867-874.

4. Ross R. Atherosclerosis: an inflammatory disease. N Engl J Med. 1999; 340(2):115-126.

5. Kotler DP. Cachexia. Ann Intern Med. 2000;133(8):622-634.

6. Johnson PM, Vogt SK, Burney MW, Muglia LJ. COX-2 inhibition attenuates anorexia during systemic inflammation without impairing cytokine production. Am J Physiol Endocrinol Metab. 2002;282(3): E650-E656.

7. Raisz LG. Physiology and pathophysiology of bone remodeling. Clin Chem. 1999;45(8 Pt 2):1353-1358.

8. Gan WQ, Man SF, Senthilselvan A, Sin DD. Association between chronic obstructive pulmonary disease and systemic inflammation: a systematic review and a meta-analysis. Thorax. 2004;59(7):574-580.

9. Barnes PJ, Celli BR. Systemic manifestations and comorbidities of COPD. Eur Respir J. 2009;33(5):1165-1185.

10. Vestbo J, Prescott E, Almdal T, et al. Body mass, fat-free body mass, and prognosis in patients with chronic obstructive pulmonary disease from a random population sample: findings from the Copenhagen City Heart Study. Am J Respir Crit Care Med. 2006;173(1):79-83.
11. Schols AM, Soeters PB, Dingemans AM, Mostert R, Frantzen PJ, Wouters EF. Prevalence and characteristics of nutritional depletion in patients with stable COPD eligible for pulmonary rehabilitation. Am Rev Respir Dis. 1993;147(5):1151-1156.

12. Celli BR, Cote CG, Marin JM, et al. The body-mass index, airflow obstruction, dyspnea, and exercise capacity index in chronic obstructive pulmonary disease. N Engl J Med. 2004;350(10):1005-1012.

13. Cruz-Jentoft AJ, Baeyens JP, Bauer JM, et al. Sarcopenia - European consensus on definition and diagnosis: report of the European Working Group on Sarcopenia in Older People. Age Ageing. 2010;39(4): 412-423.

14. Kalyani RR, Corriere M, Ferrucci L. Age-related and disease-related muscle loss: the effect of diabetes, obesity, and other diseases. Lancet Diabetes Endocrinol. 2014;2(10):819-829.

15. [No authors listed]. ATS statement: guidelines for the six-minute walk test. Am J Respir Crit Care Med. 2002;166(1):111-117.

16. [No authors listed]. Lung function testing: selection of reference values and interpretative strategies. Am Rev Respir Dis. 1991;144(5): $1202-1218$.

17. Jones SE, Maddocks M, Kon SS, et al. Sarcopenia in COPD: prevalence, clinical correlates and response to pulmonary rehabilitation. Thorax. 2015;70(3):213-218.

18. Marquis K, Debigare R, Lacasse Y, et al. Midthigh muscle crosssectional area is a better predictor of mortality than body mass index in patients with chronic obstructive pulmonary disease. Am J Respir Crit Care Med. 2002;166(6):809-813.

19. Wagner PD. Possible mechanisms underlying the development of cachexia in COPD. Eur Respir J. 2008;31(3):492-501.

20. Lee JY, Chon GR, Rhee CK, et al. Characteristics of patients with chronic obstructive pulmonary disease at the first visit to a pulmonary medical center in Korea: the Korea COPD Subgroup Study team cohort. J Korean Med Sci. 2016;31(4):553-560.

21. Swallow EB, Reyes D, Hopkinson NS, et al. Quadriceps strength predicts mortality in patients with moderate to severe chronic obstructive pulmonary disease. Thorax. 2007;62(2):115-120.

22. Burtin C, Ter Riet G, Puhan MA, et al. Handgrip weakness and mortality risk in COPD: a multicentre analysis. Thorax. 2016;71(1):86-87.

23. de Godoy I, Donahoe M, Calhoun WJ, Mancino J, Rogers RM. Elevated TNF- $\alpha$ production by peripheral blood monocytes of weight-losing COPD patients. Am J Respir Crit Care Med. 1996;153(2):633-637.

24. Eid AA, Ionescu AA, Nixon LS, et al. Inflammatory response and body composition in chronic obstructive pulmonary disease. Am J Respir Crit Care Med. 2001;164(8 Pt 1):1414-1418.

25. Schols AM. Pulmonary cachexia. Int J Cardiol. 2002;85(1):101-110.

26. Agustí AG, Noguera A, Sauleda J, Sala E, Pons J, Busquets X. Systemic effects of chronic obstructive pulmonary disease. Eur Respir J. 2003; 21(2):347-360.

27. Hole DJ, Watt GC, Davey-Smith G, Hart CL, Gillis CR, Hawthorne VM. Impaired lung function and mortality risk in men and women: findings from the Renfrew and Paisley prospective population study. BMJ. 1996; 313(7059):711-716.

28. Schünemann HJ, Dorn J, Grant BJ, Winkelstein W Jr, Trevisan M. Pulmonary function is a long-term predictor of mortality in the general population: 29-year follow-up of the Buffalo Health Study. Chest. 2000; 118(3):656-664.

29. Steiner MC. Sarcopaenia in chronic obstructive pulmonary disease. Thorax. 2007;62(2):101-103.

30. Schols AM, Buurman WA, van den Brekel AJ, Dentener MA, Wouters EF. Evidence for a relation between metabolic derangements and increased levels of inflammatory mediators in a subgroup of patients with chronic obstructive pulmonary disease. Thorax. 1996;51(8):819-824.

31. Cazzola M, Novelli G. Biomarkers in COPD. Pulm Pharmacol Ther. 2010;23(6):493-500. 
International Journal of COPD

\section{Publish your work in this journal}

The International Journal of COPD is an international, peer-reviewed journal of therapeutics and pharmacology focusing on concise rapid reporting of clinical studies and reviews in COPD. Special focus is given to the pathophysiological processes underlying the disease, intervention programs, patient focused education, and self management protocols.

This journal is indexed on PubMed Central, MedLine and CAS. The manuscript management system is completely online and includes a very quick and fair peer-review system, which is all easy to use. Visit http://www.dovepress.com/testimonials.php to read real quotes from published authors 